\title{
Syntactic Ambiguity Resolution and Task Influence by Korean Learners of English*
}

\author{
Jung Hyun Lim \\ (Konkuk University)
}

\begin{abstract}
Lim, Jung Hyun. (2017). Syntactic Ambiguity Resolution and Task Influence by Korean Learners of English. Language Research, 53.2, 321-341.
\end{abstract}

The present study investigates the questions of (a) how Korean speakers of learning English process locally ambiguous reduced relative clause during on-line comprehension and (b) whether/how task at hands changes second language (L2) processing patterns in ambiguity resolution. Participants read the locally ambiguous sentences that contain reduced relative clauses on a self-paced reading task. The stimuli sentences were manipulated with verb morphology, post-ambiguity cue, and different types of comprehension questions. The results showed that the L2 speakers were able to make use of both verb morphology and post-ambiguity cue information and that the sensitivity was influenced by the question types. The study suggests that the L2 learners are influenced by both verb morphology and cue information when resolving the ambiguity, and that reading goals significantly affects the process of ambiguity resolution. The findings are discussed in light of the good-enough (GE) language processing.

Keywords: sentence processing, good-enough processing, ambiguity resolution, task effects, reduced relative clause

\section{Introduction}

One of the overarching research questions in psycholinguistics is to understand the human language mechanisms of sentence processing and comprehension. Most of the models of language comprehension that have

* This work was supported by the KU Research Professor Program of Konkuk University. 
been put forth over the decades have assumed that we comprehend and interpret sentences correctly and accurately all the time (Frazier, 1978; Frazier and Rayner, 1982; Rayner, Carlson, and Frazier, 1983; MacDonald, Pearlmutter, and Seidenberg, 1994; Trueswell, Tanenhaus, and Garnsey, 1994). In the past years, however, there have been a series of studies that have reported miscomprehension or underspecification of some syntactic structures in native speakers of English (Christianson, Hollingworth, Halliwell, and Ferreira, 2001; Ferreira, 2003; Ferreira, Bailey, and Ferraro, 2002; Swets, Desmet, Clifton, and Ferreira, 2008). The sentences that induce either global or local ambiguity, such as garden-path sentences (Bever, 1970), main verb versus reduced relative clause (RC) ambiguity (Juffs, 1998, 2006; MacDonald, 1994), or subject-object ambiguity (Frenck-Mestre and Pynte, 1997, Juffs and Harrington, 1995), have been studied a lot in this stream of research in the field of psycholinguistics.

Over the recent decades, researchers have shown much interests in second language (L2) processing mechanisms as one of the sources that occurs the difficulty of ultimate attainment in L2 acquisition. Most of the methodologies and designs in the existing L2 studies have been largely motivated and modified from the studies in first language (L1) processing literature. One of the main research questions in the L2 processing literature is whether L2 speakers have the same processing mechanism as L1 speakers; to be more specific, whether L2 speakers make use of syntactic and semantic information during reading as similar as L1 speakers. The existing studies so far have yielded the mixed results. Some studies showed that L2 speakers are different from L1 speakers in terms of syntactic representation during on-line processing; that is, L2 learners' processing system is less-detailed than that of L1 speakers (Felser, Roberts, Gross, and Marinis, 2003; Marinis, Roberts, Felser and Clahsen, 2005; Jacob and Felser, 2015), while other studies provided the results that L2 learners displayed the same kind of sensitivity to both syntactic and semantic information during comprehension as native speakers of English (Frenck-Mestre and Pynte, 1997; Jackson, 2008; Lim and Christianson, 2013, 2015). The debate over the issue of quantitative or qualitative difference between L1 and L2 processing is still the heart of the L2 processing, and there are many issues yet to 
be solved, such as task influence, proficiency effect, working memory demands, or L1 difference.

The present study attempts to provide additional and novel data to the existing L2 processing literature by looking at whether L2 learners are able to integrate different kinds of information during processing, and by examining one of the important factors in language processing, but not much studied yet with L2 group, which is the task influence. Specifically, the study will investigate L2 speakers' ambiguity resolution of reduced relative clauses, and the modularity of task effect on ambiguity resolution. In the next section, the background studies on processing syntactic ambiguity in both L1 and L2 group, and task effects on language processing will be reviewed. Then present study will be described along with its results and discussion.

\section{Background}

When we comprehend a sentence, different kinds of linguistic information, such as syntactic, semantic and pragmatic information, need to be processed and appropriately integrated to reach at the correct interpretation. Much of the work in both L1 and L2 have investigated syntactically ambiguous sentences containing main verb versus reduced relative clause (Juffs, 1998; MacDonald, 1994; Rah and Adone, 2010; Trueswell, et al., 1994; Yang and Shin, 2013), prepositional phrase (PP) attachment (Frenck-Mestre and Pynte, 1997), or subject-object ambiguity (Ferreira and Clifton, 1986; Juffs and Harrington, 1996; Rayner et al., 1983), in order to test how people make use of those kinds of linguistic information during sentence comprehension in the field of psycholinguistics. Since the present study deals with the reduced relative clause, only the previous studies on the specific structure of interest will be reviewed in this section.

MacDonald (1994) investigated how different types of constraints converge or compete each other on syntactic ambiguity resolution of reduced relative clauses. In the three experiments, three types of constraints, verb 
argument structure frequencies, post-ambiguity non DO-phrases, and pre-ambiguity information, were examined on a self-paced reading paradigm. In Experiment 1, the frequency of verb argument structure and the post-ambiguity non DO-phrases were manipulated as in the example sentences below (1).

(1) a. The ruthless dictator/ overthrown in the coup/ was hated/ throughout the country.

b. The ruthless dictator/ captured in the coup/ was hated/ throughout the country.

c. The ruthless dictator/ fought in the coup/ was hated/ throughout the country.

d. The ruthless dictator/ overthrown just after dawn/ was hated/ throughout the country.

e. The ruthless dictator/ captured just after dawn/ was hated/ throughout the country.

f. The ruthless dictator/ fought just after dawn/ was hated/ throughout the country.

The sentence (1a) and (1d) have unambiguous verbs that are less likely to occur ambiguity because of the verb morphology; the sentence (1b) and (1e) include transitive-only verbs that easily permit the main verb interpretation; and finally the sentence (1c) and (1f) contain optionally transitive verbs which may or may not require a direct object depending on the context. The post-ambiguity not-DO phrases 'in the coup' as in (1a)-(1c) was defined as 'good cue' constraint, because the preposition 'in' usually does not introduce a direct object, such that it immediately blocks the main verb interpretation, and instead guides to the reduced relative clause interpretation. The post-ambiguity phrase like 'just after dawn' as in (1d)-(1f) was defined as 'bad cue' constraint in the experiment, since readers can easily expect a direct object to come after 'just' (e.g., just one victim) until they see the later words of the phrase 'after dawn'. This can initially allow the readers the main verb interpretation, and then require the reanalysis process towards the later sentence. The native speak- 
ers of English read those experimental sentences word-by-word on their own reading speed rate. Reading times were analyzed as a chunk identified as slashes in (1). Results on the ambiguous region (e.g., overthrown in the coup) showed that the sentence (1b) took the longest times than any other conditions, suggesting that two competing constraints- that is, activation for simpler direct object reading and the post-ambiguity cue that inhibit the direct object reading, were imposed during on-line processing. The reading times on the disambiguating region (e.g., was hated) showed that native speakers of English took longer reading times for the sentence (1f) than the other conditions, pointing out that the number of alternative argument structures and post-ambiguity cue play important roles in ambiguity resolution. Additionally, the experiment found out the reading time difference between (1a) and (1b), which indicated that the participants were also sensitive to the verb morphology itself. A series of the experiments in the study of Macdonald (1994) pointed out that language parser is dependent not solely on the syntactic information but rather on the interaction of lexical, syntactic, and semantic information (for detail information for the other two experiments, see Macdonald (1994)).

Many studies on L2 speakers have usually been motivated from the L1 studies, and it is not an exception here on the issue of ambiguity resolution. Juffs $(1998,2006)$ was motivated by MacDonald (1994), asking the similar questions with L2 speakers; to be more specific, Juffs asked the question of whether and how L2 speakers of different first language background (Chinese, Korean, Japanese, and Romance) process reduced relative clauses during on-line comprehension. Using a grammaticality judgement task and a self-paced reading task, three groups (Chinese-speaking learners, learners of SOV word order language (Japanese and Korean), learners of SVO word order language (Romance)) participated in reading sentences that contain main verb/reduced relative ambiguities. Similar to the experiments of MacDonald (1994), the verb argument structure and post-ambiguity cue information were manipulated, in order to see whether L2 learners are able to show the same degree of sensitivity to those variables as native speakers of English. The series of Juffs' studies found that (a) the non-native speakers showed the similar pattern with 
native speakers of English; in other words, the L2 learners were also being led to the garden path, especially when the verb was ambiguous transitive verb and the post-ambiguity non-DO phrases did not immediately inhibit the main verb reading, (b) the L2 learners were also able to be sensitive to the verb argument structure and the post-ambiguity cues just as native speakers, but with less robust degree and slower speed, and (c) the leaners' first language played a crucial role in accuracy and speed of processing main verb/reduced relative ambiguities, showing that the Chinese L2 learners had more difficulty with the unambiguous morphology than the other learner groups, because of the lack of past participle morphology in Chinese. The studies thus suggested that although processing patterns are similar between native processing and L2 processing, L2 processing is certainly slower than L1 processing, and that L1 typological difference from English can make a good prediction in accuracy and speed in ambiguity resolution.

As seen in Juff's $(1998,2006)$ studies, it seems that we need to consider other factors when we deal with L2 group, such as L2 proficiency or different L1 background. In fact, recent studies have pointed out that task at hands can be one of the important factors in both L1 and L2 processing (Jackson and Bobb, 2009; Leeser, Brandl, and Whiteglass, 2001; J-H Lim and Christianson, 2013, 2015; Swets et al., 2008). For example, Swets et al. (2008) manipulated the comprehension question types to investigate whether the different types of questions would influence on ambiguous relative clause attachment with native speakers of English. Sentence types (fully ambiguous, NP1 disambiguation, NP2 disambiguation) and types of comprehension questions (relative clause questions, superficial questions, and occasional questions) were manipulated to examine whether ambiguity advantages (i.e., reading ambiguous sentences faster) occurs and whether/how different types of comprehension questions influence on the parsing strategies. Example materials are presented in (2). 
(2) a. The mother of the bride who embarrassed herself at the reception was complaining to the priest. (fully ambiguous)

b. The father of the bride who embarrassed himself at the reception was complaining to the priest. (NP1 disambiguation)

c. The father of the bride who embarrassed herself at the reception was complaining to the priest. (NP2 disambiguation)

d. RC question: was the mother/bride/father embarrassed? Superficial question: Did someone complain to/compliment the priest?

Results showed that readers were significantly faster in reading the globally ambiguous sentences when asked superficial questions throughout the experiment, consistent with previous studies showing speed advantages for ambiguous sentences (Traxler, Pickering, and Clifton, 1998; van Gompel, Pickering, and Traxler, 2001). The readers did not seem to put much effort into resolving the ambiguity on the basis that there was no specific information to help them to resolve it. It was also found that reading times were much slower in globally ambiguous sentences such as (2a) than in the disambiguated sentence such as (2b) or (2c) in the RC question condition. Based on the finding that ambiguity yielded faster reading times, the authors concluded that language processing can sometimes be incomplete and ambiguous sentences are left unresolved especially when there is no information to disambiguate them and no demand for disambiguating them. Furthermore, since the task demand made reading times different, it is suggested that human language processing is strategic, being dependent on the goals of reading.

The findings in Swets et al. (2008) were discussed under the framework of the good-enough language processing, proposed by Ferreira and her colleagues (Christianson et al., 2001, 2016; Ferreira, 2003; Ferreira et al., 2002). The good-enough language processing has been proposed with substantial data through a series of studies over the recent decades. For example, Christianson et al. (2001) asked native English speakers the question 'Did Anna dress the baby?' to the sentence below in (3). 
(3) While Anna dressed the baby that was cute and cuddly played in the crib.

Surprisingly, the native English speakers incorrectly answered 'yes' to the above question. Furthermore, the participants also were $100 \%$ correct in answering to another question 'Did the baby play in the crib?' This finding suggests that people tend to misinterpret the garden path sentences, and moreover the misinterpretation lingers on their mind, even though they recover from it later. In line with this study, Ferreira (2003) found that even native English speakers misinterpreted the passive sentences significantly more often when the structure was inconsistent with the world knowledge (i.e., implausible), as in 'the dog was bitten by the man' than when the sentence was plausible as in 'the man was bitten by the dog'. Based on the findings from these series of the studies, the authors suggests the good enough language processing, in which the heuristic parser (e.g., NVV word order, or semantic/world knowledge) is 'fast and frugal' in reaching at an interpretation, and thus syntactic representations can sometimes be underspecified or overridden by the heuristically-derived interpretation. In other words, when the sentence meaning represented in a reader's mind is good-enough, regardless of its veridicality, a reader tends to comprehend the sentence in a good-enough way, possibly yielding in an incorrect final interpretation. Recently, the good-enough language processing was also observed in the data with L2 group (J-H Lim and Christianson, 2013, 2015).

\section{The Current Study}

Although the previous studies provide us with useful information to understand language processing mechanisms, there still needs more research to probe the unanswered or debatable research questions. One of the research questions that the current study seeks to answer is how Korean speakers of learning English are able to process local ambiguity of the main verb versus reduced relative clause, modified from the study of MacDonald (1994). However, the current study specifically focuses 
on the L2 learners' ability to make use of verb morphology rather than verb argument structure, since most of the previous L2 studies examined did not clearly tear verb morphology apart from verb argument structure. Also, another factor of interest in the current study is task demands on language processing, as suggested by the series of studies ( $\mathrm{J}-\mathrm{H}$ Lim and Christianson, 2013, 2015; Swets et al., 2008). Factors such as L2 proficiency and L1 difference have often been investigated in the previous studies, but none of the studies has yet looked at L2 speakers reading behaviors as a function of different comprehension question when processing ambiguous sentences, and thus this study will provide novel data to $\mathrm{L} 2$ processing literature.

\subsection{Method}

\subsubsection{Participants}

Thirty-six college students were recruited in a large university located in Seoul, Korea. The participants were all Korean native speakers who have studied English as a second language after their puberty. All were compensated with 10,000 won (KRW) for their participation.

\subsubsection{Materials}

Since the present study was motivated from the experiments by MacDonald (1994) and Juffs (1998, 2006), all stimuli were designed in a similar way as those previous studies. Three factors were manipulated; verb morphology, post-ambiguity cue, and types of comprehension questions. Sample sentences are presented as in (4).

(4) a. The short girl heard in the garden was a very pretty student.

b. The short girl heard almost every day was a very pretty student.

c. The famous scholar known by every student wrote a great book.

d. The famous scholar known just after war wrote a great book.

Two types of verbs were used, one is the verbs that have the same past and past participle forms as in (4a), such that it easily can guide to the active transitive interpretation until the main verb of the sentence comes. 
The other is the verbs that have different forms between past and past participle just like (4c), which rather immediately inhibit the active transitive meaning before readers arrives at the main verb, given that the participants have knowledge of verb morphology in English. Being consistent with the study of MacDonald (1994), the good and bad constraints were implemented in order to test whether and how much L2 learners are sensitive to post-ambiguity cue information. The post-ambiguity phrase, consisting of non-DO phrase (e.g., in the garden, or almost every day), can either facilitate or inhibit the active transitive interpretation. In the good cue condition, the active transitive interpretation can be immediately inhibited as in (4a), leading to the reduced relative interpretation, whereas in the bad cue condition as in (4b), the inhibition delays longer, such that it takes more time to resolve the local ambiguity. Lastly but importantly, the types of comprehension questions were manipulated to see whether the ambiguity processing is modulated by the task at hands. The comprehension questions types were three kinds; RC type, superficial type, and occasional type. The RC type of question asked whether participants correctly interpret reduced relative clause; for example, "was the short girl heard?" to the trial sentences (4a) and (4b). The superficial type of question probed participants' overall understanding of the whole sentence, but at a very superficial level, such as "was the girl a teacher?". In the third condition, the same kind of superficial question as in the second condition appears, but on an occasional base; that is, one or two randomly chosen out of 12 to 15 trials.

The two factors of verb morphology and post-ambiguity cue were manipulated as within-subjects, and the factor of comprehension question types was designed as between-subjects. There were total 40 experimental sentences; 20 of which include ambiguous verbs that have the same past and past participle forms and the rest 20 of which include unambiguous verbs that have different past and past participle forms. The stimuli sentences were distributed across two lists in a Latin square design such that each participant saw every item in only one of the two within-subjects factor conditions. With the between-subjects factor (three comprehension types), total 6 lists were yielded. Each list also contained 96 filler sentences 
with various structures which did not contain any of reduced relative clauses. The fillers in RC and superficial comprehension question conditions were followed by the comprehension question for every sentence, whereas the fillers in occasional comprehension question condition appeared occasionally in the same way as the stimuli condition.

\subsubsection{Procedures}

Participants performed the experiment individually on the computer, programmed with E-prime professional software version 2.0. The experimenter verbally explained the procedure of the experiment to the participants, and they were able to read the written instruction on the computer screen as well. A set of stimuli sentences and filler sentences appeared on the monitor. The participants were asked to read each sentence at their normal reading pace by pressing the space bar to continue the sentence on a self-paced, word-by-word, non-cumulative reading task. Comprehension questions were followed by every sentence except in the occasional question condition. Participants pressed one of the buttons indicating a "yes" or "no" answer, and did not receive any feedback on the answer. Practice session was provided to make the subject be familiar with the task, and there was a short break session on the half way through the experiment. The total experiment was completed in about 40-45 minutes.

\subsubsection{Results}

Comprehension accuracy was overall $91 \%$ of the time on the filler sentences, ensuring that the participants were attentive to reading during the experiment. Reading times were analyzed on all stimuli sentences. In the previous studies (Juffs, 1998; Macdonald, 1994), reading times were analyzed as three chunks: (a) ambiguous composed of ambiguous $\mathrm{RC}$ verb and the good/bad cue, (b) disambiguation composed of the main verb and the one word following the verb, and (c) end composed of the rest of the sentence. Although such analysis gave us a big picture of the participants' processing profile, it would be more precise to analyze word-by-word to investigate how people read along the sentence. Thus, in the current study, I defined six critical regions, and analyzed all of them. The critical regions of interests in analysis were (a) ambiguous 
RC verb, (b) cue, (c) main verb (mv), (d) one word following the main verb $(\mathrm{mv}+1)$, and $(\mathrm{e})$ the second word after the main verb $(\mathrm{mv}+2)$, and (f) the rest of the sentence (rest). The critical regions (a) and (b) together can be regarded as the ambiguous region, and (c) and (d) together in this study can be regarded as the disambiguation region in the analysis on MacDonald (1994). Analyzing separate regions unlike the previous studies would provide us with more accurate information in terms of timing on L2 learners' ambiguity resolution of the reduced RC sentences, if any effect is observed. The sample example is presented below in (5).

(5) The short girl /heard /in the garden /was /a/ very/ pretty student.
(a)
(b)
(c) (d) (e)

(f)

Reading times were analyzed using a mixed-effects linear model in program R (Baayen, Davidson, and Bates, 2008). Using a stepwise selection procedure, the factors of verb morphology, cue and question type were included as fixed effects in the final model. Random intercepts for participants and items were also included and some of random slopes contributed to the model in several critical regions. The random effects structure was fitted using likelihood ratio tests. Here only the fixed and random effects and interactions that were significant $(p \leq .05)$ were reported in the result tables. Based on the analyses of the critical regions, the reading times of the ambiguous RC verb (a) and the main verb (c) did not yield any significant results in any of the conditions.

Table 1 presents the results of the mixed model analysis of the reading times of the cue region in different conditions.

Table 1. Fixed Effects in the Linear Mixed-effects Model of Reading Times in the cue region

\begin{tabular}{ccccc}
\hline Predictor & Estimate & SE & $t$-value & $p$ \\
\hline \hline Intercept & 1724.24 & 84.63 & 20.37 &.$<001^{* * *}$ \\
\hline cue & 108.95 & 36.88 & 2.95 & $.005^{* *}$ \\
\hline verb $^{*}$ cue* ${ }^{*}$-type & -42.70 & 19.23 & -2.22 & $.02^{*}$ \\
\hline
\end{tabular}

Note. verb $=$ verb morphology; cue $=$ post-ambiguity cue; q-type $=$ question type 
As presented in Table 1, there were the significant main effect of cue and the interaction among all three factors. Generally, readers took significantly more time to read sentences with the bad cue than with the good cue, regardless of the comprehension question types. Since there were all three factor interactions $(p=.02)$, post-hoc analyses were conducted based on the different question types in order to reveal the pattern of the interaction between the factors- verb morphology, cue, and the question type.

Using a mixed-effects linear model, reading times of the cue region were separately analyzed in three question types, including the other two factors of verb morphology and cue. As a result of the post-hoc analyses, it turned out that the main effect of cue was significant in all types of questions; however, the interaction between verb morphology and cue reached at a significant level $(p=.02)$ only in the RC question type. In the RC question type, whereas reading times were slightly slower with the good-cue than with the bad-cue for the ambiguous verbs, reading times were much slower with the bad-cue than with the good-cue for the unambiguous verbs.

As mentioned earlier, there was no significant effect observed in the region of main verb. More reliable effects were found at the one word following the main verb, which is termed as the region, mv+1. Table 2 shows the statistical result of the fixed-effects linear-mixed model in the $m+1$ region.

Table 2. Fixed Effects in the Linear Mixed-effects Model of Reading Times at the $m v+1$

\begin{tabular}{ccccc}
\hline Predictor & Estimate & SE & $t$-value & $p$ \\
\hline \hline Intercept & 505.05 & 22.52 & 22.43 &.$<001^{* * *}$ \\
\hline verb & -26.69 & 13.69 & -1.94 & .05. \\
\hline verb* $^{*}$ cue $^{*}$-type & 20.96 & 8.19 & 2.56 & $.01^{*}$ \\
\hline
\end{tabular}

Note. verb = verb morphology; cue $=$ post-ambiguity cue; q-type $=$ question type

As presented in Table 2 above, the variable of verb morphology and the interaction among the three factors yielded significant effects. The significant main effect of verb morphology (although it is just marginal $p=.05$ ) 
indicated that people generally tended to read the sentences with the ambiguous verbs much slower than the sentences with the unambiguous verbs, reflecting the garden-path effect and the recovery processes of reduced relative clauses. In order to figure out the structure of the interaction among the three variables, post-hoc tests using a mixed-effects linear model were performed. The post-hoc results yielded different significant effects depending on the types of comprehension questions. To be more specific, (a) main effect of verb morphology(marginally significant at $p=.05$ ) was only observed in the RC question type condition, (b) the interaction of verb morphology and cue was significant only in the occasional question type condition, and (c) there was no effect in the superficial question condition.

At the second word following the main verb $(m v+2)$, the similar statistical effects as in the $\mathrm{mv}+1$ region were observed; the verb morphology yielded the significant main effect $(p=.01)$ and the interaction among the three factors were marginally significant at $p=.05$. As a result of the post-hoc analysis on the interaction among the three factors, it showed that the effect was different depending on the comprehension question type, similar to the result of the $m v+1$ region. In the rest of the sentence, the main effects of cue $(p=.02)$ and question type $(p=.05)$, and the marginal effect of interaction between cue and question type ( $p=.08)$ were observed. The L2 participants read the sentences involving bad cues much slower than the sentences with good cues in the wrap-up region, and they had much difficulty with the RC question than the other two question conditions. The interaction between cue and question type indicated that the main effect of cue was observed $(p=.02)$ only in the RC question type, not in the other two question conditions, pointing out the different depth of processing involved depending on the different question types, being consistent with the previous study, Swets et al. (2008). 


\section{Discussion}

The present study asked the research questions of (a) whether the Korean learners of English are influenced by the verb morphology and post-verb ambiguity cue information in resolution of locally ambiguous reduced relative clauses (RC), and (b) how the different types of comprehension questions affect the depth of processing sentences in L2 speakers. To seek the answers to the questions, the materials were designed in the similar way as the previous studies of MacDonald (1994) and Juffs (1998). The investigation of the sensitivity to the verb morphology and the cue information was expected to provide us the information on the processing mechanisms internalized in L2 readers. In addition, the manipulation of the depth of comprehension questions would allow us to see whether people change their reading strategy and patterns depending on the tasks at hands.

The current study yielded several interesting findings on the L2 speakers' reading behaviors, and has implications for language processing as well. First, the L2 participants were influenced by both verb morphology and post-ambiguity cue information, when they were faced with the sentences containing the reduced RC. The L2 speakers noticed whether the cue was good or bad right in the cue region, which consists of the non-DO phrase (e.g., in the garden or almost every day). They read the sentences with bad cue much slower than the ones with the good cue, as shown in the main effect of cue $(p<.01)$ in the cue region, indicating the L2 participants' abilities to differentiate the kinds of cue information. The sensitivity to the verb morphology appeared at the one word following the main verb, so-called $m v+1$ region. In the $m v+1$ region, the main effect of verb morphology $(p=.05)$ indicated that people took more time to read the sentences with ambiguous verb (such as heard, called, or caught) than the sentences with unambiguous verb (such as known, eaten, or seen). This result exactly showed that the L2 readers initially comprehended the reduced RC verb as the main verb of the sentence, especially when the verb has the same form in past and past participle, i.e., 'ambiguous verb' in this study. Then, they needed to re-compute the incorrect analysis 
as they saw the real main verb of the sentence later on, resulting in the longer reading times with the ambiguous verb. The unambiguous verb reduced this reanalysis process, such that it yielded the shorter reading times than the ambiguous verb. This result is consistent with the previous studies on L1 native speakers (MacDonald, 1994) and on L2 speakers (Juffs, 1998; Rah and Adone, 2010; Yang and Shin, 2013).

Second, the interactions among the factors of verb morphology, cue, and comprehension question type were observed in several critical regions; the cue region, $\mathrm{mv}+1, \mathrm{mv}+2$ and the rest regions. These interactions indicate that the influence of each variable on the ambiguity resolution of the reduced RC is varied dependent of other variables. All these interactions point toward the one important implication on sentence processing; that is, the task effect on the ambiguity resolution of reduced relative clause by L 2 readers. The results in the current study are in line with the previous studies that showed different reading performances depending on the task types (Jackson and Bobb, 2009; Leeser et al., 2001; J-H Lim and Christianson, 2013, 2015; Swets et al., 2008). As with the previous studies, the current study also revealed that the patterns of L2 reading was contingent upon the different comprehension question types. The most attempt to resolve the temporary ambiguity caused by the reduced relative clause was captured in the condition where the L2 participants were asked about the content of the relative clause itself. In the RC comprehension question type, the main effects of verb morphology and the interaction between the two factors observed in the critical regions of cue, $\mathrm{mv}+1$, and $\mathrm{mv}+2$ confirmed that the $\mathrm{L} 2$ readers tried to inspect the disambiguating region with more care when they misinterpreted the reduced $\mathrm{RC}$ verb as the main verb at an initial stage. In this process, the post-ambiguity cue information also affected the reading times, yielding the interaction between the two factors. If the different task would not have affected processing of ambiguity resolution in on-line comprehension, the reading times would have been similar regardless of different question types.

Just as the native speakers' reading behavior in the relative clause attachment in different types of comprehension question types in the study of Swets et al. (2007), the current study provides the same line of data with 
the L2 group to the language processing literature. As reviewed earlier, in Swets et al. (2008) experiments, the native speakers tended not to resolve the local ambiguity of the relative clause attachment, leaving syntactic underspecification in the interpretation of the relative clause, when the question was superficial and occasional. The authors thus suggested that language processing is goal-dependent and strategic, which is explained under the good enough processing approach (Christianson et al., 2001, 2016; Ferreira, 2003; Ferreira et al., 2002). The good enough (GE) approach to language processing suggests that the language processor is "fast and frugal" (Ferreira, 2003) and is "bounded" under a various constraints, such as task demands, processing load, and time (Christianson, $2016 ; 820)$. The results in the current study are completely supporting for the GE language processing. When the questions specifically asked about the relative clause, the L2 speakers spent longer reading time on the disambiguating region and displayed more sensitivity to the linguistic input, i.e., both verb morphology and cue information, whereas when the questions ask just at a superficial level, their attempts to resolve the ambiguity were much lessen and even delayed to the later region.

While the current study yielded many interesting findings on the L2 processing mechanisms, there are things that need to be addressed in the near future studies. First, the regions that the L2 participants showed sensitivity to the critical factors in this study were different from the previous studies (MacDonald, 1994; Rah and Adone, 2010). The sources of the different outcomes may be owing to the different setting of the critical regions among the studies, or to the different population (native English group for MacDonald (1994) vs. L2 group for the current study). In the current study, the critical region was defined as word-by-word, providing more accurate data in terms of processing timing, while the previous studies analyzed the ambiguous region as a chunk consisting of the reduced RC verb and the non-DO cue phrase. The current study did not find any effect at the reduced RC verb itself whereas the previous studies found the effect of verb morphology and cue information in the ambiguous region, which included the reduced RC verb. The future study with native speakers of English on the current setting may reveal the 
source of the different outcomes. In addition, the present study did not control for the transitivity of the verb, since the focus of the study was on the sensitivity to the verb morphology. The future study on the manipulation of the transitivity of the verb along with verb morphology would provide more information on the use of verb argument structure in the ambiguity resolution by $\mathrm{L} 2$ learners.

\section{Conclusion}

The present study found that Korean speakers of learning English were able to make use of both the verb morphology and post-verb ambiguity cue information. In addition, importantly, the processing patterns were significantly fluctuated by task types at hands; readers' attention to linguistic input and attempts to resolve the ambiguity became stronger when the question was about the reduced RC. Altogether, the findings of the current study give favorable data to the good enough language processing in L2 processing mechanisms. As noted earlier, we need to consider other variables with L2 group to understand L2 language processing. Thus, although this study shed much light on ambiguity resolution process by L2 learners of English, future research will require to examine L2 proficiency, typological difference between L1 and L2, and/or working memory capacity, with various other sentence structures.

\section{References}

Baayen, R. H., Davidson, D. J., and Bates, D. M. (2008). Mixed-effects modeling with crossed random effects for subjects and items. Journal of Memory and Language 59, 390-412.

Bever, T. G. (1970). The cognitive basis for linguistic structure. In J. R. Hayes, ed., Cognition and the development of language, 279-362. New York: Wiley.

Christianson, K. (2016). When language comprehension goes wrong for the right reasons: Good Enough, underspecified, or shallow language processing. The Quarterly Journal of Experimental Psychology 69, 817-828. 
Christianson, K., Hollingworth, A., Halliwell, J. F., and Ferreira, F. (2001). Thematic roles assigned along the garden path linger. Cognitive Psychology 42, 368-407.

Felser, C., Roberts, L., Gross, R., and Marinis, T. (2003). The processing of ambiguous sentences by first and second language learners of English. Applied Psycholinguistics 24, 453-489.

Ferreira, F. (2003). The misinterpretation of noncanonical sentences. Cognitive Psychology 47, 164-20.

Ferreira, F., Bailey, K., and Ferraro, V. (2002). Good enough representations in language comprehension. Current Directions in Psychological Science 11, 11-15.

Ferreira, F. and Clifton, C., Jr. (1986). Independence of syntactic processing. Journal of Memory and Language 25, 348-368.

Frazier, L. (1978). Sentence processing: Review. In M. Coltheart, ed., Attention and performance: Vol. XI. The psychology of reading, 559-586. Hillsdale, NJ: Erlbaum.

Frazier, L. and Fodor, J. D. (1978). The sausage machine: A new two-stage parsing model. Cognition 6, 291-325.

Frazier, L. and Rayner, K. (1982). Making and correcting errors during sentence comprehension: Eye movements in the analysis of structurally ambiguous sentences. Cognitive Psychology 14, 178-210.

Frenck-Mestre, C. and Pynte, J. (1997). Syntactic ambiguity resolution while reading in second and native languages. Quarterly Journal of Experimental Psychology 50A, 119-148.

Jackson, C. (2008). Proficiency level and the interaction of lexical and morphosyntactic information during L2 sentence processing. Language Learning 58.4, 875-909.

Jackson, C. and Bobb, S. C. (2009). The processing and comprehension of wh-questions among second language speakers of German. Applied Psycholinguistics 30, 603-636.

Jacob, G. and Felser, C. (2015). Reanalysis and semantic persistence in native and non-native garden-path recovery. The Quarterly Journal of Experimental Psychology 69.5, 907-925.

Juffs, A. (1998). Main verb vs reduced relative clause ambiguity resolution in second language sentence processing. Language Learning 48, 107-147.

(2006). Processing reduced relative versus main verb ambiguity in English as a second language: A replication study with working memory. In R.Slabakova, S. Montrul, and P. Prevost, eds., Inquiries in linguistic development in honor of Lydia White, 213-234. Amsterdam: Benjamins. 
Juffs, A. and Harrington, M. (1995). Parsing effects in second language sentence processing: Subject and object asymmetries in wh-extraction. Studies in Second Language Acquisition 17, 483-516.

Lim, J.-H. and Christianson, K. (2013). Second language sentence processing in reading for comprehension and translation. Bilingualism: Language and Cognition 16.3, 518-537.

Lim, J.-H. and Christianson, K. (2015). Second language sensitivity to agreement errors: Evidence from eye movements during comprehension and translation. Applied Psycholinguistics 36.6, 1283-1315.

Leeser, M. J., Brandl, A., and Whiteglass, C. (2011). Task effects in second language processing research. In P. Tromovich and K. McDonough, eds., Applied priming methods to L2 learning, teaching and research: Insights from psycholinguistics, 179-198. Amsterdam: Benjamins.

MacDonald, M. C. (1994). Probabilistic constraints and syntactic ambiguity resolution. Language and Cognitive Processes 9, 121-136.

MacDonald, M. C., Pearlmutter, N. J., and Seidenberg, M. S. (1994). Lexical nature of syntactic ambiguity resolution. Psychological Review 101, 676-703.

Marinis, T., Roberts, L., Felser, C., and Clahsen, H. (2005). Gaps in second language sentence processing. Studies in Second Language Acquisition 27, 53-78.

Rah, A. and Adone, D. (2010). Processing of the reduced relative clause versus main verb ambiguity in L2 learners at different proficiency levels. Studies in Second Language Acquisition 32, 79-109.

Rayner, K., Carlson, M., and Frazier, L. (1983). The interaction of syntax and semantics during sentence processing: Eye movements in the analysis of semantically biased sentences. Journal of Verbal Learning and Verbal behavior 22, 358-374.

Swets, B., Timothy, D. Clifton, C., and Ferreira, F. (2008). Undersepcification of syntactic ambiguities: Evidence from self-paced reading. Memory \& Cognition 36.1, 201-216.

Tranxler, M. J., Pickering, M. J., and Clifton, C., Jr. (1998). Adjunt attachment is not a form of lexical ambiguity resolution. Journal of Memory and Language 39, 558-592.

Truesewell, J. C., Tanenhaus, M. K., and Garnsey, S. M. (1994). Semantic influences on parsing: Use of thematic role information in syntactic ambiguity resolution. Journal of Memory and Language 33, 285-318.

Van Gompel, R. P. G., Pickering, M. J., and Traxler, M. J. (2001). Reanalysis in sentence processing: Evidence against current constraint-based and two-stage models. Journal of Memory and Language 45, 225-258.

Yang, P.-L. and Shin, S.-C. (2013). A reading-time study of the main verb versus 
reduced relative clause ambiguity resolution by English learners in Taiwan. Applied Psycholinguistics 34, 1109-1133.

Jung Hyun Lim

Research Institute for Multilingualism and Multiculturalism Konkuk University

120 Neungdong-ro, Gwangjin-gu, Seoul

E-mail: junghlim@konkuk.ac.kr

Received: June 30, 2017

Revised version received: August 14, 2017

Accepted: August 24, 2017 
